\title{
Discontinuing noninvasive ventilation in severe chronic obstructive pulmonary disease exacerbations: a randomised controlled trial
}

\author{
Jacobo Sellares ${ }^{1,2}$, Miquel Ferrer ${ }^{1,2}$, Antonio Anton ${ }^{3}$, Hugo Loureiro ${ }^{1,2}$, \\ Carolina Bencosme ${ }^{1,4}$, Rodrigo Alonso ${ }^{5}$, Pilar Martinez-Olondris 6,7 , \\ Javier Sayas ${ }^{5}$, Patricia Peñacoba ${ }^{3}$ and Antoni Torres ${ }^{1,2}$
}

Affiliations: 'Servei de Pneumologia, Institut Clínic de Respiratori, Hospital Clínic, Institut d'Investigacions Biomediques August Pi i Sunyer (IDIBAPS), Barcelona, Spain. ${ }^{2}$ Centro de Investigación Biomedica En RedEnfermedades Respiratorias, (CibeRes, CB06/06/0028) Instituto de Salud Carlos III. Madrid, Spain. ${ }^{3}$ Dept of Pneumology, Hospital de Sant Pau, Barcelona, Spain. ${ }^{4}$ Hospital General de la Plaza de la Salud, Santo Domingo, Dominican Republic. ${ }^{5}$ Servicio de Neumologia, Hospital 12 de Octubre, Madrid, Spain. ${ }^{6}$ Servicio de Neumologia, Hospital del Mar, Barcelona, Spain. ${ }^{7}$ Hospital Plato, Barcelona, Spain.

Correspondence: Miquel Ferrer, UVIR, Servei de Pneumología, Hospital Clínic, Villarroel 170. 08036 Barcelona, Spain. E-mail: miferrer@clinic.ub.es

@ERSpublications

NIV can be directly discontinued when a COPD exacerbation is resolved and patients tolerate unassisted breathing http://ow.ly/fJCY30bYfme

Cite this article as: Sellares J, Ferrer M, Anton A, et al. Discontinuing noninvasive ventilation in severe chronic obstructive pulmonary disease exacerbations: a randomised controlled trial. Eur Respir J 2017; 50: 1601448 [https://doi.org/10.1183/13993003.01448-2016].

ABSTRACT We assessed whether prolongation of nocturnal noninvasive ventilation (NIV) after recovery from acute hypercapnic respiratory failure (AHRF) in chronic obstructive pulmonary disease (COPD) patients with NIV could prevent subsequent relapse of AHRF.

A randomised controlled trial was performed in 120 COPD patients without previous domiciliary ventilation, admitted for AHRF and treated with NIV. When the episode was resolved and patients tolerated unassisted breathing for $4 \mathrm{~h}$, they were randomly allocated to receive three additional nights of NIV $(n=61)$ or direct NIV discontinuation $(n=59)$. The primary outcome was relapse of AHRF within 8 days after NIV discontinuation.

Except for a shorter median (interquartile range) intermediate respiratory care unit (IRCU) stay in the direct discontinuation group $(4(2-6)$ versus $5(4-7)$ days, $\mathrm{p}=0.036)$, no differences were observed in relapse of AHRF after NIV discontinuation (10 (17\%) versus 8 (13\%) for the direct discontinuation and nocturnal NIV groups, respectively, $\mathrm{p}=0.56$ ), long-term ventilator dependence, hospital stay, and 6-month hospital readmission or survival.

Prolongation of nocturnal NIV after recovery from an AHRF episode does not prevent subsequent relapse of AHRF in COPD patients without previous domiciliary ventilation, and results in longer IRCU stay. Consequently, NIV can be directly discontinued when the episode is resolved and patients tolerate unassisted breathing.

Received: July 202016 | Accepted after revision: March 302017

Trial registration: ClinicalTrials.gov identifier: NCT00775710

Support statement: 2009-SGR-911, IDIBAPS, ICREA academia 2013, CibeRes (CB06/06/0028)-ISCiii, FUCAP. Funding information for this article has been deposited with the Crossref Funder Registry.

Conflict of interest: None declared.

Copyright OERS 2017 


\section{Introduction}

Noninvasive ventilation (NIV) is an established indication in patients with acute exacerbations of chronic obstructive pulmonary disease (COPD) and acute hypercapnic respiratory failure (AHRF) with respiratory acidosis [1,2]. Although different studies have defined factors predicting the outcome of NIV in terms of intubation and mortality in patients with severe AHRF [3-6], these studies did not assess the best method to discontinue NIV.

When an episode of AHRF is resolved, the British Thoracic Society guidelines recommend reducing the periods of ventilation according to clinical criteria, reducing diurnal ventilation before nocturnal to avoid relapse of respiratory failure [7]. However, this recommendation was not supported by prospectively controlled clinical studies.

A retrospective study in patients successfully treated with NIV for an episode of severe AHRF showed that the degree of respiratory acidosis at admission and a noninfectious cause of AHRF were associated with long-term dependency to NIV, with a higher incidence in non-COPD than in COPD patients [8]. Unlike the extensive body of knowledge in weaning from invasive mechanical ventilation [9], this study highlighted the lack of standardised protocols for discontinuing NIV and the need for prospective validation of a weaning protocol in patients managed with NIV for an episode of AHRF. Moreover, this study did not assess the influence of the method to discontinue NIV in the incidence of long-term dependency of NIV.

A recent pilot study in patients with COPD exacerbation recovering from an episode of AHRF did not show any benefits for either stepwise or immediate withdrawal of NIV [10]. This study, in a limited number of patients, may have been underpowered to detect differences, as recognised by the authors, who stated that larger prospective studies are required.

In patients with COPD exacerbations who have recovered from an episode of AHRF that required NIV, it is not clear whether keeping nocturnal NIV for several days after episode resolution would avoid subsequent relapse of respiratory failure with respiratory acidosis and the development of long-term dependency of NIV or not. We therefore compared the effectiveness of keeping ventilation for three nights with direct discontinuation after recovery from the episode of AHRF in reducing the incidence of new episodes of severe AHRF.

\section{Methods}

Patients

A prospective, randomised controlled study was conducted in the Intermediate Respiratory Care Units (IRCUs) from the Departments of Pneumology at the Hospital Clinic, Hospital del Mar and Hospital de Sant Pau in Barcelona, and Hospital 12 de Octubre in Madrid, Spain. Patients admitted with an episode of AHRF because of COPD exacerbation, defined as arterial carbon dioxide tension $\left(\mathrm{PaCO}_{2}\right)>45 \mathrm{mmHg}$ and arterial $\mathrm{pH}<7.35$ after standard medical treatment had been initiated, who had received NIV and had recovered from the acute episode with adequate tolerance of unassisted breathing were considered eligible. We defined COPD according to the GOLD definition [11]. Patients with facial or cranial trauma or surgery, recent gastric, oral or oesophageal surgery, tracheostomy or other upper airway disorders, active upper gastrointestinal bleeding, and with an excessive amount of respiratory secretions or weak cough, had not been considered for NIV treatment. Exclusion criteria were 1) previous use of domiciliary NIV; and 2) uncooperative state with inability to understand or unwillingness to follow the protocol's instructions. The Ethics Committees of the institutions approved the study and written informed consent was obtained from patients.

\section{Procedures}

Indications for NIV had been established by the attending physicians. All instrumentation is part of the routine clinical practice of patients treated with NIV in an acute care setting. The criteria used for implementing NIV were 1) moderate-to-severe dyspnoea accompanied by a respiratory rate $\geqslant 25$ breaths $\cdot \min ^{-1}$, or signs of increased breathing effort, such as active contraction of the accessory respiratory muscles; and 2) AHRF as defined above.

Noninvasive ventilation was applied with specific NIV ventilators (BiPAP ST-D and VISION Ventilator, Respironics, Inc., Murrysville, PA, USA, and VPAP ST and Elysee 150, ResMed, San Diego, CA, USA) using bi-level positive airway pressure modes as previously described [12-14]. Face masks were used as first choice, but nasal masks were an option if patients did not tolerate a face mask. The initial inspiratory positive airway pressure was set at $12 \mathrm{cmH}_{2} \mathrm{O}$ and the level was raised by 2 to $3 \mathrm{cmH}_{2} \mathrm{O}$ as tolerated, but never exceeded $25 \mathrm{cmH}_{2} \mathrm{O}$. The initial expiratory positive airway pressure was set at $5 \mathrm{cmH}_{2} \mathrm{O}$, and the level was raised by 1 to $2 \mathrm{cmH}_{2} \mathrm{O}$ if needed to improve hypoxaemia or comfort. The inspiratory oxygen 
fraction $\left(\mathrm{FiO}_{2}\right)$ was set to achieve arterial $\mathrm{O}_{2}$ saturation by pulse oximetry $\left(\mathrm{SpO}_{2}\right)$ above $90 \%$ or arterial oxygen tension $\left(\mathrm{PaO}_{2}\right)$ above $60 \mathrm{mmHg}$. Arterial blood gas samples were obtained from each patient before and after connecting to the ventilator, and subsequently as clinically indicated. Noninvasive ventilation alternated with unassisted breathing according to patients' needs and tolerance; the periods on NIV were gradually reduced if patients tolerated longer periods on unassisted breathing.

After clinical stabilisation and with the cause of AHRF under control in eligible patients, arterial blood gases were taken early in the morning while on NIV. If arterial $\mathrm{pH} \geqslant 7.35$, we proceeded to discontinue NIV [7]. Patients were assessed after $4 \mathrm{~h}$ of unassisted breathing with Venturi oxygen, at the minimal concentration to achieve the same oxygenation targets of NIV. If patients did not worsen clinically and arterial blood gases showed hypercapnia without respiratory acidosis $\left(\mathrm{PaCO}_{2}>45 \mathrm{mmHg}\right.$ and arterial $\mathrm{pH}$ $\geqslant 7.35)$, then they were randomly allocated (1:1 ratio, computer-generated sequence) into two groups: nocturnal NIV and direct discontinuation of NIV. Otherwise, NIV was resumed and patients were assessed on the following day. Concealment was done with sequentially numbered opaque sealed envelopes, which were opened by the investigator only after the informed consent was obtained. Patients and staff were aware of treatment allocations owing to the nature of the interventions.

\section{Direct discontinuation group}

Patients allocated to this group received the conventional Venturi oxygen mask. The setting of $\mathrm{FiO}_{2}$ was the same as used before randomisation. Oxygen therapy was administered for as long as patients needed; if the oxygenation target could be achieved without supplemental oxygen, then patients could breathe room air.

\section{Nocturnal NIV group}

Patients allocated to this group prolonged the use of NIV for three additional nights during sleep time. The same conditions of NIV were maintained during the study. Nocturnal NIV was applied for as long as possible, but periods on unassisted breathing were allowed according to the patients' tolerance and to administer nebulised bronchodilators. After the third night, NIV was discontinued. Patients received daytime conventional Venturi oxygen during the 3 days of nocturnal NIV and continuously afterwards using the same conditions as in the direct discontinuation group. If patients from both groups developed relapse of AHRF, NIV was restarted and discontinuation of NIV was reassessed the following day. A similar procedure was done if subsequent episodes of relapse of AHRF occurred.

\section{Data collection}

All relevant data were collected at admission and at randomisation from the medical records and bedside flow charts including laboratory, radiologic and microbiologic information. We calculated the Acute Physiology And Chronic Health Evaluation (APACHE-II) Score [15]. Patients were followed until hospital discharge, death or up to 6 months after randomisation.

\section{Definitions}

Relapse of AHRF within 8 days after randomisation was the primary end-point, defined as the presence of at least two of the following: 1) respiratory acidosis (arterial $\mathrm{pH}<7.35$ together with $\mathrm{PaCO}_{2}>45 \mathrm{mmHg}$ ); 2) $\mathrm{SpO}_{2}<90 \%$ or $\mathrm{PaO}_{2}<60 \mathrm{mmHg}$ at $\mathrm{FiO}_{2} \geqslant 0.50$; and persistence for $30 \mathrm{~min}$ or more of either 3) respiratory frequency $>35$ breaths $\mathrm{min}^{-1}$; 4) decreased consciousness, agitation or diaphoresis; or 5) clinical signs suggestive of respiratory muscle fatigue or increased work of breathing, such as use of respiratory accessory muscles, paradoxical motion of the abdomen or retraction of intercostal spaces $[16,17]$.

In the absence of previous decisions to limit life-support measures, patients were immediately intubated if any of the following predefined major clinical events occurred: respiratory or cardiac arrest; respiratory pauses with loss of consciousness or gasping for air; psychomotor agitation inadequately controlled by sedation; massive aspiration; persistent inability to remove respiratory secretions; heart rate $<50$ beats. $\min ^{-1}$ with loss of alertness; and severe haemodynamic instability without response to fluids and vasoactive drugs [16-18].

Successful discontinuation of NIV was considered when no episodes of relapse of AHRF were observed for 8 days following NIV discontinuation. A secondary outcome was long-term dependency of NIV, when all of the following criteria were present: 1 ) stable resolution of the triggering factor; 2) inability to stop NIV for at least 8 consecutive days (after at least two attempts) because of worsening clinical status, a rise in $\mathrm{PaCO}_{2}$ with respiratory acidosis (arterial $\mathrm{pH}<7.35$ ) and/or recurrent AHRF without any identifiable cause; and 3) discharge home with domiciliary NIV [8]. Other secondary end-points were length of stay, IRCU and hospital mortality, survival at 6 months and hospital readmission at 6 months among hospital survivors. 


\section{Statistical analysis}

Sample size calculation

The primary end-point was relapse of AHRF after NIV discontinuation. Previous data concerning the rate of this outcome were lacking at the onset of this trial. Although this is not exactly the same outcome, we rely on the incidence of late NIV failure in a previous study [19]. We expected a $23 \%$ incidence of relapse of AHRF in the direct discontinuation group. We considered that reducing this outcome to $10 \%$ in the nocturnal NIV group would be clinically relevant. Therefore, we estimated a minimal sample size of 124 patients (62 in each group), with alpha error 0.05 and $\beta$-error 0.20 .

Comparisons between the two groups

Qualitative or categorical variables were compared with the Chi-squared or Fisher's exact tests, when appropriate. Quantitative continuous variables were compared using the Mann-Whitney nonparametric test; median (interquartile range) values are reported. The Kaplan-Meier estimate-of-survival curve was used to determine the cumulative 180-day survival probability; survival curves between the two groups were compared using the log-rank test. The analyses were intention-to-treat, and the level of significance was set at 0.05 (two-tailed). Data were processed with IBM SPSS Statistics Version 20.

\section{Results}

\section{Patients}

Among 168 consecutively registered patients, we studied 120 patients in two periods, between December 2007 and July 2009, and between December 2013 and December 2014 (figure 1): 59 were randomly allocated to the direct discontinuation group and 61 to the nocturnal NIV group. Baseline characteristics and physiological variables of patients are summarised in tables 1,2 and 3. All patients were current or former smokers. Both groups did not differ significantly at baseline regarding comorbidities, the main cause of COPD exacerbation that required NIV and severity at admission and of hypercapnic respiratory failure. The $\mathrm{PaO}_{2}$ after $24 \mathrm{~h}$ with NIV treatment was slightly higher in patients from the nocturnal NIV group (table 2).

NIV was discontinued after the first attempt in 106 (88\%) patients. The proportion of patients who required two or more attempts was higher in the direct discontinuation group, but the median duration of NIV before randomisation was similar between both groups (table 3). The physiological parameters at the time of randomisation while patients were breathing spontaneously were also similar between both groups.

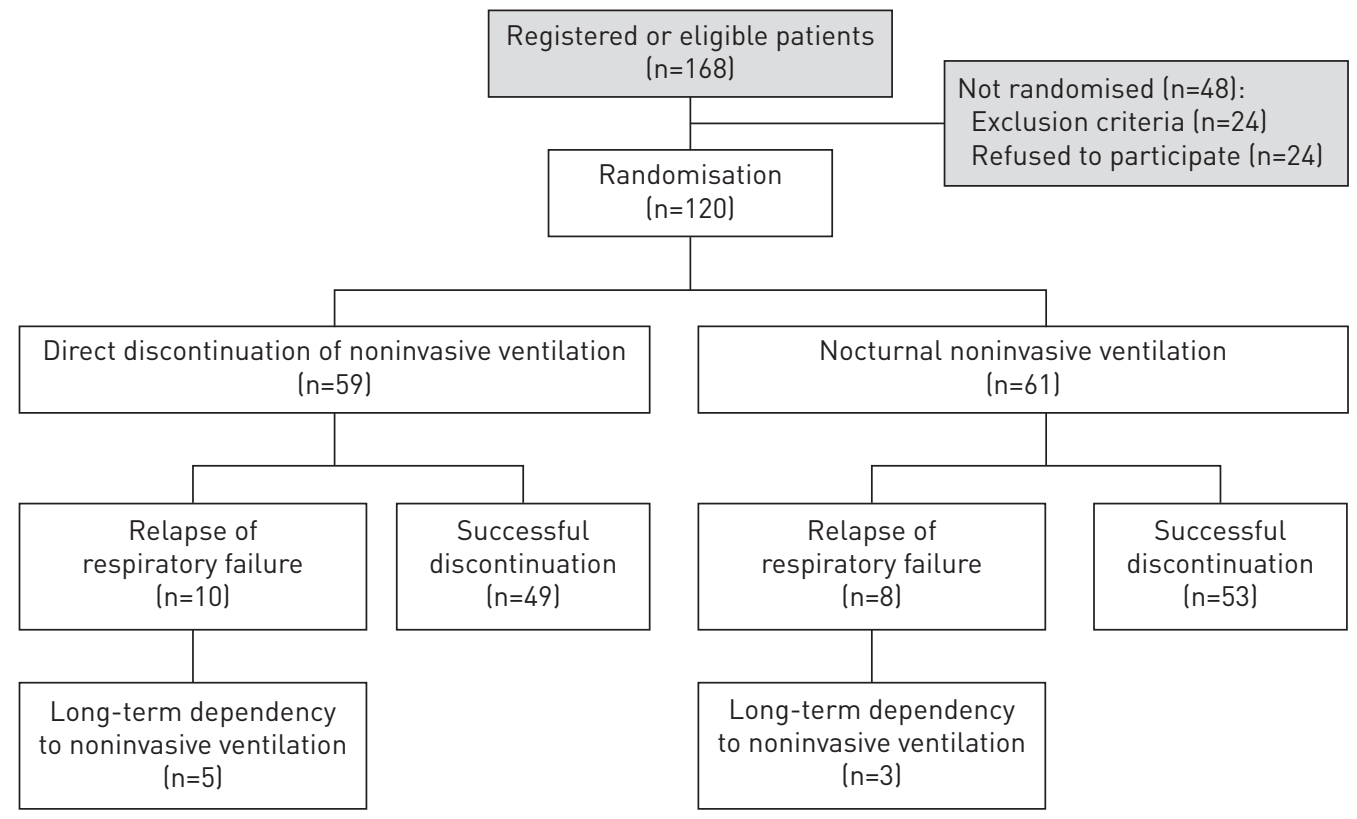

FIGURE 1 Trial profile. Exclusion criteria were previous indication of domiciliary noninvasive ventilation ( $n=15)$, and uncooperative state with inability to understand or unwillingness to follow the protocol's instructions $(n=9)$. 


\begin{tabular}{|c|c|c|c|}
\hline Variable & Direct discontinuation ( $n=59$ ) & Nocturnal NIV (n=61) & p-value \\
\hline Age years & $71(61-78)$ & $71(61-78)$ & 0.87 \\
\hline Male/female & $39 / 20$ & $41 / 20$ & 0.90 \\
\hline FEV1 \% pred ${ }^{\#}$ & $35(28-45)$ & $38(30-49)$ & 0.33 \\
\hline \multicolumn{4}{|l|}{ Other comorbidities } \\
\hline Chronic heart disorders" & $24(41)$ & 24 (39) & 0.88 \\
\hline Immunosuppression ${ }^{+}$ & 2 (3) & $5(8)$ & 0.44 \\
\hline Diabetes mellitus & $11(19)$ & $13(21)$ & 0.72 \\
\hline Solid cancer & $2(3)$ & $7(12)$ & 0.093 \\
\hline Chronic liver disorders & $3(5)$ & $6(10)$ & 0.32 \\
\hline Chronic renal failure & $0(0)$ & 2 (3) & 0.16 \\
\hline Morbid obesity ${ }^{\S}$ & $9(15)$ & $13(21)$ & 0.39 \\
\hline Main cause of COPD exacerbation & & & 0.52 \\
\hline Infection & $32(54)$ & $28(46)$ & \\
\hline Undetermined & $24(41)$ & $31(51)$ & \\
\hline Cardiac & $3(5)$ & $2(3)$ & \\
\hline APACHE-II on admission & $18(14-20)$ & $17(15-21)$ & 0.81 \\
\hline
\end{tabular}

Data are presented as $\mathrm{n}, \mathrm{n}(\%)$ or median (interquartile range). NIV: noninvasive ventilation; FEV1: forced expiratory volume in $1 \mathrm{~s}$; COPD: chronic obstructive pulmonary disease; APACHE II: Acute Physiology and Chronic Health Evaluation II. " : this information was available in 38 patients from the direct discontinuation group and 48 from the nocturnal NIV group; ๆ: chronic heart disorders include coronary artery disease, hypertensive or valvular heart diseases, and dilated myocardial disease of any cause; ${ }^{+}$: immunosuppression includes neutropenia after chemotherapy or bone marrow transplant, drug-induced immunosuppression in solid organ transplant or as a result of corticosteroids or cytotoxic therapy, and human immunodeficiency virus-related disorders; $\$$ : morbid obesity (body mass index $\geqslant 40 \mathrm{~kg} \cdot \mathrm{m}^{-2}$ ) information was obtained from the patients' medical records and/or the most recent forced spirometry reports.

\section{Treatment compliance and complications detected after randomisation}

In the nocturnal NIV group, all patients received NIV for the first two nights, except one patient prematurely discontinued after presenting with upper gastrointestinal bleeding. Five patients declined to continue on NIV during the third night (table 4).

The rate of complications after patients had been randomised was similar between both groups.

\section{Length of stay and outcome variables (table 4)}

The length of IRCU stay was significantly longer in patients from the nocturnal NIV group $(\mathrm{p}<0.001)$. However, the hospital stay was not significantly different between both groups (table 4).

The rate of relapse of AHRF with the need to restart NIV (10 (17\%) versus eight (13\%) for the direct discontinuation and nocturnal NIV groups, respectively, $\mathrm{p}=0.56$, mean difference $4 \%, 95 \%$ CI -9 to $17 \%$ ) and the time from randomisation to relapse were similar between both groups. One patient from the nocturnal NIV group needed intubation after relapse of AHRF. The rate of long-term dependency on NIV, transfer to long-term care units, hospital mortality and 6-month survival (figure 2) were also similar between both groups, as well as the hospital readmission within 6 months among survivors from the initial episode (figure 3 ).

To avoid potential confusion related to the inclusion of patients with morbid obesity, we have done a sub-analysis in patients without morbid obesity, with similar rates of relapse of AHRF (8 (16\%) versus 7 (15\%) for the direct discontinuation and nocturnal NIV groups, respectively, $\mathrm{p}=0.85)$.

\section{Discussion}

In these selected COPD patients with good initial response to NIV, nocturnal NIV for three nights after the initial recovery from an episode of AHRF resulted in longer IRCU stay without any clinically significant benefit compared with direct discontinuation of NIV.

Weaning from invasive ventilation has been extensively studied in the literature and was the topic of an International Consensus Conference [9]. In contrast, there is a paucity of information in defining a strategy to discontinue NIV, and, indeed, the optimal method to discontinue NIV after the recovery of an episode of AHRF has not been established yet. 
TABLE 2 Physiological parameters of patients before randomisation

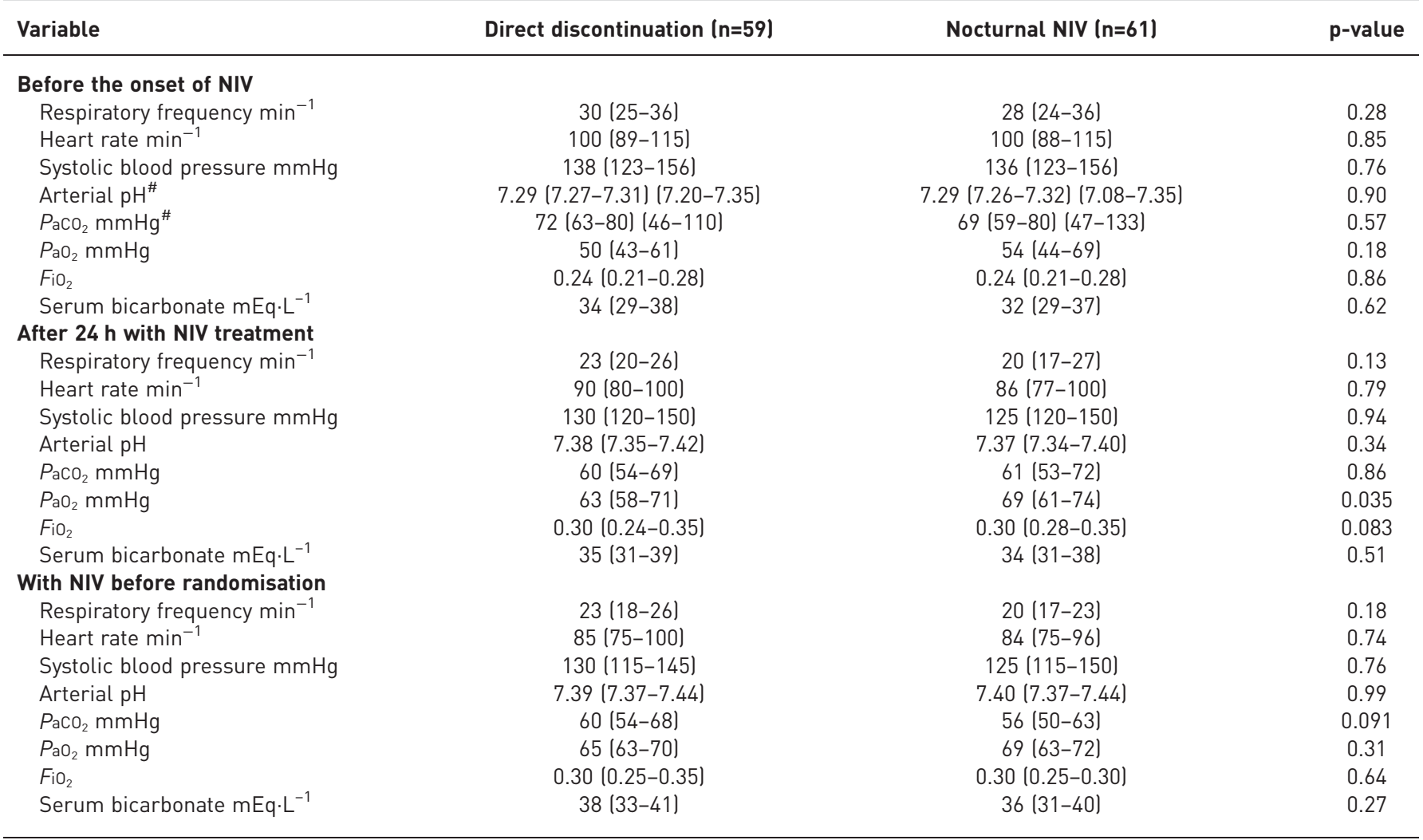

Data are presented as median (interquartile range). ${ }^{\#}$ : ranges also indicated. NIV: noninvasive ventilation; $P_{a O_{2}}$ : arterial carbon dioxide tension; $\mathrm{PaO}_{2}$ : arterial oxygen tension; $\mathrm{FiO}_{2}$ : inspiratory oxygen fraction.

Clinical improvement and stability of the patient's condition are the most important factors in determining when NIV can be safely discontinued. It is often recognised by patients who decide independently to stop using the machine [7]. There is a marked variability in the methods used to discontinue NIV among published series that reflects the absence of a prospectively validated protocol.

\begin{tabular}{|c|c|c|c|}
\hline & Direct discontinuation ( $n=59$ ) & Nocturnal NIV (n=61) & p-value \\
\hline Prior duration of NIV days & $2(1-3)$ & $2(1-4)$ & 0.38 \\
\hline NIV discontinuation at & & & 0.040 \\
\hline First attempt & 48 & 58 & \\
\hline Second attempt & 11 & 2 & \\
\hline Third attempt & 0 & 1 & \\
\hline \multicolumn{4}{|c|}{$\begin{array}{l}\text { Physiological variables on unassisted } \\
\text { breathing }\end{array}$} \\
\hline Respiratory frequency $\min ^{-1}$ & $22(19-24)$ & $20(19-24)$ & 0.064 \\
\hline Heart rate $\min ^{-1}$ & $88(80-100)$ & $85(76-93)$ & 0.40 \\
\hline Systolic blood pressure mmHg & 125 (115-139) & $120(112-132)$ & 0.18 \\
\hline $\mathrm{FiO}_{2}$ & $0.28(0.24-0.31)$ & $0.28(0.24-0.30)$ & 0.76 \\
\hline Arterial pH & $7.40(7.38-7.44)$ & $7.41(7.38-7.43)$ & 0.92 \\
\hline $\mathrm{PaCO}_{2} \mathrm{mmHg}$ & $58(51-66)$ & 55 (52-59) & 0.16 \\
\hline $\mathrm{PaO}_{2} \mathrm{mmHg}$ & $62(58-70)$ & $64(59-67)$ & 0.62 \\
\hline Serum bicarbonate $\mathrm{mEq} \cdot \mathrm{L}^{-1}$ & $36(33-40)$ & $34(32-37)$ & 0.11 \\
\hline
\end{tabular}

Data are presented as median (interquartile range) or $\mathrm{n}$. NIV: noninvasive ventilation; $\mathrm{PaCO}_{2}$ : arterial carbon dioxide tension; $\mathrm{PaO}_{2}$ : arterial oxygen tension; $\mathrm{FiO}_{2}$ : inspiratory oxygen fraction. 


\begin{tabular}{|c|c|c|c|}
\hline Variable & Direct discontinuation $(n=59)$ & Nocturnal NIV (n=61) & p-value \\
\hline Facial skin lesions & 4 (7) & $3(5)$ & 0.66 \\
\hline Gastric distension & 0 & $1(2)$ & $>0.99$ \\
\hline Upper gastrointestinal bleeding & 0 & $1(2)^{\S}$ & $>0.99$ \\
\hline Nosocomial pneumonia & $1(2)$ & 0 & $>0.99$ \\
\hline Urinary tract infection & $1(2)$ & 0 & 0.31 \\
\hline IRCU stay days & $4(2-6)$ & $5(4-7)$ & 0.036 \\
\hline Hospital stay days & $12(9-15)$ & $13(10-17)$ & 0.21 \\
\hline Relapse of AHRF & $10(17)$ & $8(13)$ & 0.56 \\
\hline Time from randomisation to relapse $\mathrm{h}^{\#}$ & $34(23-84)$ & $24(6-93)$ & 0.50 \\
\hline Patients intubated ${ }^{\#}$ & 0 & $1(13)$ & 0.25 \\
\hline Main reason for relapse of $\mathrm{AHRF}^{+}$ & & & 0.25 \\
\hline Respiratory acidosis & 6 & 8 & \\
\hline Persistent hypoxaemia at $\mathrm{FiO}_{2} \geqslant 0.50$ & 2 & 0 & \\
\hline Decreased consciousness & 1 & 0 & \\
\hline Paradoxical motion of the abdomen & 1 & 0 & \\
\hline Status 8 days after randomisation & & & 0.67 \\
\hline Successful discontinuation of NIV & $53(90)$ & 56 (92) & \\
\hline Long-term dependency of NIV & $5(9)$ & $3(5)$ & \\
\hline Death & $1(2)$ & $1(2)$ & \\
\hline Intubated & $0(0)$ & $1(2)$ & \\
\hline Drop out from the study & 2 (3) & 2 (3) & 0.97 \\
\hline Transfer to long-term care unit & 2 (3) & $3(5)$ & 0.68 \\
\hline Readmission rate at 6 months $\pi$ & $19(35)$ & $23(40)$ & 0.63 \\
\hline IRCU mortality & $1(2)$ & $1(2)$ & 0.98 \\
\hline Hospital mortality & $3(5)$ & $3(5)$ & 0.97 \\
\hline \multicolumn{4}{|l|}{ Causes of death within 6 months } \\
\hline Progressive respiratory failure & $4(7)$ & $5(8)$ & \\
\hline Multiple organ failure & $0(0)$ & $1(2)$ & \\
\hline Congestive heart failure & $0(0)$ & $1(2)$ & \\
\hline Cardiac arrest & $0(0)$ & $1(2)$ & \\
\hline Unknown & $2(3)$ & $0(0)$ & \\
\hline
\end{tabular}

Data are presented as $\mathrm{n}, \mathrm{n}(\%)$ or median (interquartile range). NIV: noninvasive ventilation; AHRF: acute hypercapnic respiratory failure; IRCU: intermediate respiratory care unit; $\mathrm{FiO}_{2}$ : inspiratory oxygen fraction. \#: among patients with relapse of AHRF; " : among survivors of the initial episode; ${ }^{+}$: the criteria for relapse of AHRF are defined in the methods section; ${ }^{\S}$ : this patient was prematurely discontinued from NIV support.

Observational and controlled clinical trials in hypercapnic patients show different methods for discontinuing NIV. These methods include a gradual withdrawal of the level and time on NIV [8, 10, 1922], reducing diurnal ventilation before nocturnal [23], predefined algorithms [24], fixed periods of time followed by direct removal $[25,26]$ and leaving the decision to the investigators' or clinicians' criteria [27, 28]. In some trials there is no mention on how NIV was removed [29-31].

During sleep, several patients with COPD present periods of decreased arterial oxygen saturation related to episodes of hypoventilation, particularly during REM sleep [32-34]. For this reason, it was recommended that NIV should be reduced gradually and removed during daytime while ventilatory support is maintained during night [7]. Such a protocol was used in a descriptive series of 64 patients with acute exacerbation of COPD that had achieved clinical stability [35]. In this study, no patients had to be reconnected to NIV or required invasive ventilation during the hospital stay.

In spite of this theoretical rationale, the efficacy of this strategy has been previously assessed only in a pilot controlled clinical trial [10]. In this study, stepwise withdrawal of NIV was done by a gradual reduction of the time on NIV for 3 days following randomisation, whereas immediate withdrawal of NIV was carried out in the same manner as in our study. Similarly, these authors found no differences in successful withdrawal of NIV or mortality between both strategies. However this study followed patients for 2 days for the primary end-point and until hospital discharge for mortality. Therefore, some cases with later adverse outcomes might have been missed.

The results of our study show that keeping night ventilation for several days after the initial resolution of an episode of AHRF did not prevent relapse of AHRF, the primary end-point, or the development of 


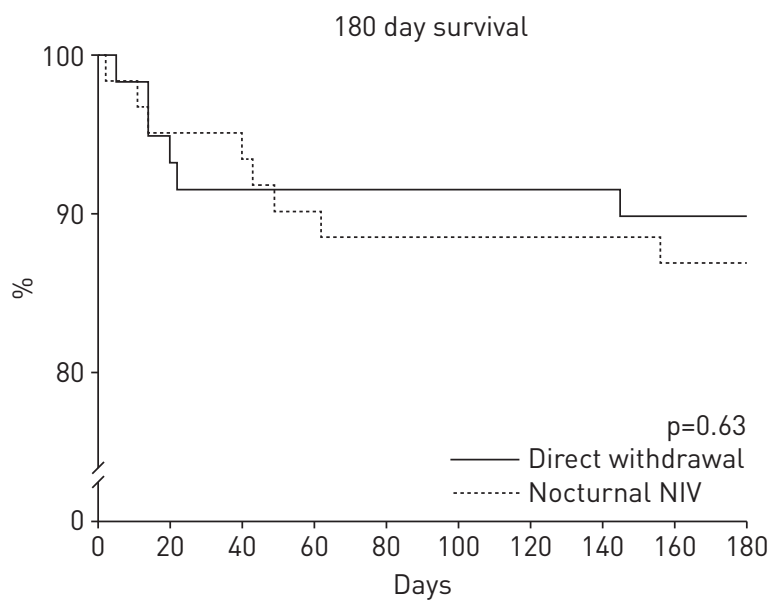

Patients remaining under the study

FIGURE 2 Survival after 180 days of entry into the protocol in both groups. NIV: noninvasive ventilation.

$\begin{array}{lccccccc}\text { Time (days) } & 0 & 30 & 60 & 90 & 120 & 150 & 180 \\ \text { Direct discontinuation } & 59 & 54 & 54 & 54 & 54 & 53 & 53 \\ \text { Nocturnal NIV } & 61 & 58 & 55 & 54 & 54 & 54 & 53\end{array}$

long-term dependency on NIV, and prolonged the IRCU stay unnecessarily. Relapse of AHRF in our study was not substantially different than the rate of late NIV failure observed previously in COPD patients [19]. In this study, late NIV failure was defined as a sudden or progressive worsening of arterial blood gases, dyspnoea and/or sensory deterioration after at least $48 \mathrm{~h}$ of starting NIV, which does not fit exactly with our primary end-point variable. By contrast, we observed a lower rate of long-term dependency on NIV than previous publications $[8,36]$. Several reasons may explain this finding. First, we enrolled COPD patients who had initially recovered from an acute episode of AHRF, and a lower rate of long-term dependency on NIV can be expected. Second, COPD, compared with non-COPD patients, was associated with less long-term dependency of NIV in a previous study [8], further explaining the low rate of this outcome in our population. Similarly, survival and hospital readmission after 6 months of the initial episode were not modified by the addition of nocturnal NIV after patients' recovery. Again, the low mortality and hospital readmission rates observed in this study may be explained by the selection of

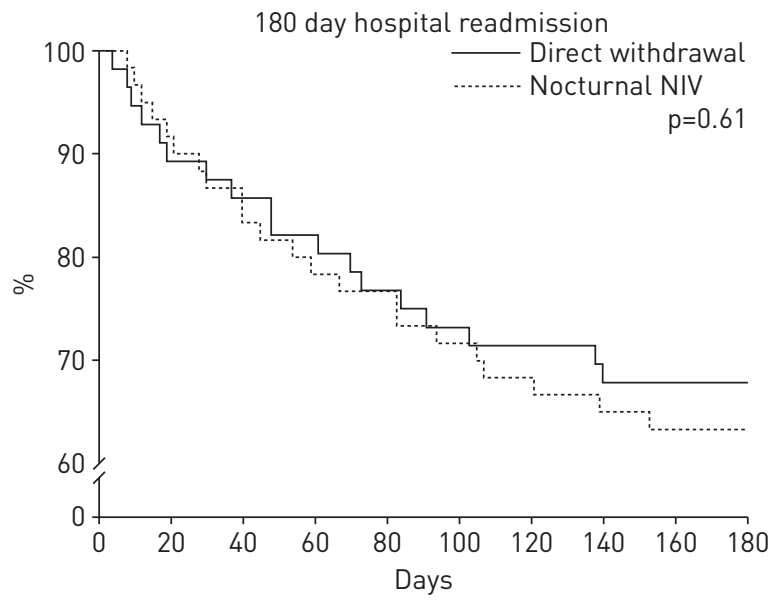

Patients remaining under the study

$\begin{array}{lccccccc}\text { Time (days) } & 0 & 30 & 60 & 90 & 120 & 150 & 180 \\ \text { Direct discontinuation } & 59 & 53 & 49 & 45 & 43 & 41 & 40 \\ \text { Nocturnal NIV } & 61 & 54 & 49 & 46 & 43 & 41 & 38\end{array}$

FIGURE 3 Patients surviving the acute episode and remaining without hospital readmission after 180 days of entry into the protocol in both groups. NIV: noninvasive ventilation. 
patients who had recovered from the initial episode of AHRF that indicated the use of NIV, and by the gradual approach used to reduce the periods on NIV according to patients' evolution.

We followed patients during 8 days for the short-term outcomes, and during 6 months for readmission or survival. Therefore, some cases with later adverse outcomes may have been missed in the previous study because of the shorter follow-up period [10].

Several limitations have to be addressed. First, we selected patients without previous indication for domiciliary ventilation, since in these patients nocturnal NIV is mandatory after the resolution of the episode of AHRF. Therefore, we cannot apply these results to patients with domiciliary NIV. This selection may have contributed to the slightly lower rate of relapse of AHRF than that expected in the nocturnal NIV group, and therefore the study may have been underpowered for this primary outcome. Second, a relevant proportion of patients had morbid obesity associated with COPD, a condition recently associated with better outcomes for NIV than non-obese COPD patients [14]. However, the lack of differences between groups persisted when patients with morbid obesity were excluded from the analysis. In addition, morbid obesity information was taken from medical records, including reports of forced spirometry manoeuvres when available for recent dates. We cannot discount that patients with morbid obesity associated with COPD could benefit from night ventilation. However, because of the small number of these patients, no conclusions can be drawn about possible long-term efficacy of NIV in them. Third, the difficulty for a correct blinding of the investigators, attending physicians and patients, a common bias in this type of open clinical trials. Despite the fact that we predefined the criteria for all relevant interventions, clinical decisions and outcome variables, this bias could not be entirely controlled. Blinding the control group with low levels of continuous positive airway pressure (CPAP) would probably result in significant bias, since mask CPAP is expected to decrease the work of breathing in these patients [37]. Other authors have used a sham NIV device for blinding patients and investigators [26]. Fourth, we chose $4 \mathrm{~h}$ as the time period to assess patients' unassisted breathing tolerance, but other authors assessed this issue after 8-12 $\mathrm{h}$ [8]. Indeed, relapse of AHRF occurred $4 \mathrm{~h}$ after randomisation in one patient from our trial; this patient would not have been enrolled using this longer time period. Fifth, there were more patients in the direct discontinuation group with two or more unsuccessful discontinuations from NIV than in the nocturnal NIV group. This potential bias could have favoured the nocturnal NIV group. Sixth, the rate of relapse of AHRF was slightly lower than that predicted, which may have decreased the statistical power of the study. Finally, although we could not demonstrate a benefit of three additional nights of NIV, it is possible that other strategies could perform as well as or even better than the strategy used in the direct discontinuation group.

In conclusion, the prolongation of NIV for three nights after recovery from an episode of AHRF does not prevent subsequent relapse of AHRF, hospital readmission or mortality in COPD patients without previous domiciliary ventilation, and results in longer IRCU stay. Consequently NIV can be directly discontinued in these patients when the acute episode is resolved and they tolerate unassisted breathing.

\section{Acknowledgements}

Miquel Ferrer is the guarantor of, and takes responsibility for the content of the manuscript, including the data and analysis. He had full access to all the data in the study and had final responsibility for the decision to submit for publication. We are indebted to all medical and nursing colleagues from the participating units for their assistance and cooperation in this study.

Roles of the authors: J. Sellares and M. Ferrer participated in the study design, recruitment of patients, data collection, analysis and interpretation of data and writing of the manuscript. A. Anton participated in the recruitment of patients, data collection and interpretation and critical revision of the manuscript. H. Loureiro, C. Bencosme, R. Alonso, P. Martinez-Olondris, J. Sayas and P. Peñacoba participated in the recruitment of patients and data collection. A. Torres participated in the study design, interpretation of data and critical review and writing of the manuscript. All the authors have read and approved the final version of the manuscript.

\section{References}

1 Lightowler JV, Wedzicha JA, Elliott MW, et al. Non-invasive positive pressure ventilation to treat respiratory failure resulting from exacerbations of chronic obstructive pulmonary disease: Cochrane systematic review and meta-analysis. BMJ 2003; 326: 185-189.

2 Ram FS, Picot J, Lightowler J, et al. Non-invasive positive pressure ventilation for treatment of respiratory failure due to exacerbations of chronic obstructive pulmonary disease. Cochrane Database Syst Rev 2004; CD004104.

3 Soo Hoo GW, Santiago S, Williams J. Nasal mechanical ventilation for hypercapnic respiratory failure in chronic obstructive pulmonary disease: determinants of success and failure. Crit Care Med 1994; 27: 417-434.

4 Ambrosino N, Foglio K, Rubini F, et al. Non-invasive mechanical ventilation in acute respiratory failure due to chronic obstructive pulmonary disease: correlates for success. Thorax 1995; 50: 755-757.

5 Antón A, Güell R, Gómez J, et al. Predicting the result of noninvasive ventilation in severe acute exacerbations of patients with chronic airflow limitation. Chest 2000; 117: 828-833.

6 Confalonieri M, Garuti G, Cattaruzza MS, et al. A chart of failure risk for noninvasive ventilation in patients with COPD exacerbation. Eur Respir J 2005; 25: 348-355. 
7 British Thoracic Society Standards of Care Committee. Non-invasive ventilation in acute respiratory failure Thorax 2002; 57: 192-211.

8 Cuvelier A, Viacroze C, Benichou J, et al. Dependency on mask ventilation after acute respiratory failure in the intermediate care unit. Eur Respir J 2005; 26: 289-297.

9 Boles JM, Bion J, Connors A, et al. Weaning from mechanical ventilation. Eur Respir J 2007; 29: 1033-1056.

10 Lun CT, Chan VL, Leung WS, et al. A pilot randomized study comparing two methods of non-invasive ventilation withdrawal after acute respiratory failure in chronic obstructive pulmonary disease. Respirology 2013; 18: 814-819.

11 Vestbo J, Hurd SS, Agustí AG, et al. Global strategy for the diagnosis, management, and prevention of chronic obstructive pulmonary disease: GOLD Executive Summary. Am J Respir Crit Care Med 2013; 187: 347-365.

12 Ferrer $\mathrm{M}$, Esquinas $\mathrm{A}$, Leon $\mathrm{M}$, et al. Noninvasive ventilation in severe hypoxemic respiratory failure: A randomized clinical trial. Am J Respir Crit Care Med 2003; 168: 1438-1444.

13 Carrillo A, Gonzalez-Diaz G, Ferrer M, et al. Non-invasive ventilation in community-acquired pneumonia and severe acute respiratory failure. Intensive Care Med 2012; 38: 458-466.

14 Carrillo A, Ferrer M, Gonzalez-Diaz G, et al. Noninvasive ventilation in acute hypercapnic respiratory failure caused by obesity hypoventilation syndrome and chronic obstructive pulmonary disease. Am J Respir Crit Care Med 2012; 186: 1279-1285.

15 Knaus WA, Draper EA, Wagner DP, et al. APACHE II: a severity of disease classification system. Crit Care Med 1985; 13: 818-829.

16 Ferrer M, Valencia M, Nicolas JM, et al. Early noninvasive ventilation averts extubation failure in patients at risk: a randomized trial. Am J Respir Crit Care Med 2006; 173: 164-170.

17 Ferrer M, Sellarés J, Valencia M, et al. Non-invasive ventilation after extubation in hypercapnic patients with chronic respiratory disorders: randomised controlled trial. Lancet 2009; 374: 1082-1088.

18 Ferrer M, Esquinas A, Arancibia F, et al. Noninvasive ventilation during persistent weaning failure: a randomized controlled trial. Am J Respir Crit Care Med 2003; 168: 70-76.

19 Moretti M, Cilione C, Tampieri A, et al. Incidence and causes of non-invasive mechanical ventilation failure after initial success. Thorax 2000; 55: 819-825.

20 Celikel T, Sungur M, Ceyhan B, et al. Comparison of noninvasive positive pressure ventilation with standard medical therapy in hypercapnic acute respiratory failure. Chest 1998; 114: 1636-1642.

21 Conti G, Antonelli M, Navalesi P, et al. Noninvasive vs. conventional mechanical ventilation in patients with chronic obstructive pulmonary disease after failure of medical treatment in the ward: a randomized trial. Intensive Care Med 2002; 28: 1701-1707.

22 Gonzalez Diaz G, Carrillo Alcaraz A, Pardo Talavera JC, et al. Noninvasive positive-pressure ventilation to treat hypercapnic coma secondary to respiratory failure. Chest 2005; 127: 952-960.

23 Brown JS, Meecham Jones DJ, Mikelsons C, et al. Using nasal intermittent positive pressure ventilation on a general respiratory ward. J R Coll Physicians Lond 1998; 32: 219-224.

24 Kramer N, Meyer T, Meharg J, et al. Randomized, prospective trial of noninvasive positive pressure ventilation in acute respiratory failure. Am J Respir Crit Care Med 1995; 151: 1799-1806.

25 Barbé F, Togores B, Rubí M, et al. Noninvasive ventilatory support does not facilitate recovery from acute respiratory failure in chronic obstructive pulmonary disease. Eur Respir J 1996; 9: 1240-1245.

26 Carrera M, Marin JM, Anton A, et al. A controlled trial of noninvasive ventilation for chronic obstructive pulmonary disease exacerbations. J Crit Care 2009; 24: 473-414.

27 Brochard L, Mancebo J, Wysocki M, et al. Noninvasive ventilation for acute exacerbations of chronic obstructive pulmonary disease. N Engl J Med 1995; 333: 817-822.

28 Maggiore SM, Richard JC, Abroug F, et al. A multicenter, randomized trial of noninvasive ventilation with helium-oxygen mixture in exacerbations of chronic obstructive lung disease. Crit Care Med 2010; 38: 145-151.

29 Plant PK, Owen JL, Elliott MW. Early use of non-invasive ventilation for acute exacerbations of chronic obstructive pulmonary disease on general respiratory wards: a multicentre randomised controlled trial. Lancet 2000; 355: 1931-1935.

30 Gay PC, Hess DR, Hill NS. Noninvasive proportional assist ventilation for acute respiratory insufficiency. Comparison with pressure support ventilation. Am J Respir Crit Care Med 2001; 164: 1606-1611.

31 Thys F, Roeseler J, Reynaert $\mathrm{M}$, et al. Noninvasive ventilation for acute respiratory failure: a prospective randomised placebo-controlled trial. Eur Respir J 2002; 20: 545-555.

32 Calverley PMA, Brezinova V, Douglas NJ, et al. The effect of oxygenation on sleep quality in chronic bronchitis and emphysema. Am Rev Respir Dis 1982; 126: 206-210.

33 Hudgel DW, Martin RJ, Capehart M, et al. Contribution of hypoventilation to sleep oxygen desaturation in chronic obstructive pulmonary disease. J Appl Physiol 1983; 55: 669-677.

34 Catterall JR, Douglas NJ, Calverley PMA, et al. Transient hypoxemia during sleep in chronic obstructive pulmonary disease is not a sleep apnea syndrome. Am Rev Respir Dis 1983; 128: 24-29.

35 Damas C, Andrade C, Araujo JP, et al. [Weaning from non-invasive positive pressure ventilation: experience with progressive periods of withdraw]. Rev Port Pneumol 2008; 14: 49-53.

36 Confalonieri M, Gorini M, Ambrosino N, et al. Respiratory intensive care units in Italy: a national census and prospective cohort study. Thorax 2001; 56: 373-378.

37 Appendini L, Patessio A, Zanaboni S, et al. Physiologic effects of positive end-expiratory pressure and mask pressure support during exacerbations of chronic obstructive pulmonary disease. Am J Respir Crit Care Med 1994; 149: $1069-1076$ 